\title{
MODELLING OF DROPLET HEATING, EVAPORATION AND BREAK-UP: RECENT DEVELOPMENTS
}

\author{
S Sazhin ${ }^{1}$, S Martynov ${ }^{1}$, I Shishkova ${ }^{2}$, C Crua ${ }^{1}$, K Karimi $^{1}$, M Gorokhovski ${ }^{3}$, E Sazhina ${ }^{1}$, \\ M Heikal $^{1}$ \\ ${ }^{1}$ School of Engineering, Faculty of Science and Engineering, University of Brighton, Cockcroft \\ Building, Brighton BN2 4GJ, UK \\ ${ }^{2}$ Low Temperature Departments, Centre of High Technologies, Moscow Power Engineering \\ Institute, Krasnokazarmennaya, 14, Moscow 111250, Russia \\ ${ }^{3}$ Univertity of Rouen, Site Universitaire du Madrillet - BP12, 76801 Saint Etienne du Rouvray \\ Cedex, France
}

\begin{abstract}
Several new approaches to the modelling of liquid droplet heating and evaporation by convection and radiation from the surrounding hot gas are reviewed. The finite thermal conductivity of the liquid, recirculation within droplets, time dependence of gas temperature and the convection heat transfer coefficient are taken into account. The relatively small contribution of thermal radiation to droplet heating allows us to describe it by a simplified model, which does not consider the variation of radiation absorption inside the droplets. In the case of stationary droplets a coupled solution of the heat conduction equation for gas and liquid phases is obtained. A transient modification of Newton's law is introduced via a correction to either the gas temperature or convection heat transfer coefficient. The solution is analysed using values of parameters relevant to liquid fuel droplet heating in a diesel engine. Since gas diffusivity in this case is more than an order of magnitude larger than liquid diffusivity, for practical applications in computational fluid dynamics (CFD) codes, this model can be simplified by assuming that droplet surface temperature is fixed. Moreover, if the initial stage of droplet heating (a few $\mu$ s) can be ignored then the steady-state solution for the gas phase can be applied for the analysis of droplet heating. This solution is described in terms of the steady-state convection heat transfer coefficient. All transient effects in this case are accounted for by liquid phase models. A decomposition technique for the solution of the system of ODEs, based on the geometrical version of the integral manifold method, is described. A comparative analysis of hydrodynamic and kinetic approaches to the problem of diesel fuel droplet evaporation is described. The kinetic approaches are based on a simplified analysis of the Boltzmann equation and its direct numerical solution. Kinetic models predict longer evaporation times and higher droplet temperature compared with the hydrodynamic model. It is recommended that kinetic effects are taken into account when modelling the evaporation process of diesel fuel droplets in realistic internal combustion engines. The preliminary results predicted by deterministic and stochastic models of droplet break-up, both implemented into the KIVA-2 code, are compared with high-speed video images of diesel sprays.
\end{abstract}

\section{Nomenclature}

$\begin{array}{llll}a, b & \text { coefficients in Eq. (1) } & P & \text { radiative power density } \\ \text { Fo } & \text { Fourier number } & R & \text { distance from the droplet centre } \\ h & \text { convection heat transfer coefficient } & R_{d} & \text { droplet radius } \\ \text { Kn } & \text { Knudsen number } & R_{d 0} & \text { initial droplet radius }\end{array}$




$\begin{array}{ll}t & \text { time } \\ T_{\text {cr }} & \text { critical temperature } \\ T_{\text {ext }} & \text { external temperature } \\ T_{g} & \text { gas temperature }\end{array}$

$\beta \quad$ evaporation coefficient

$\theta_{R} \quad$ radiative temperature

$\sigma \quad$ Stefan-Boltzmann constant

\section{Introduction}

The focus of this paper is on a summary of recent developments in the models for droplet heating and evaporation, and presentation of some new preliminary results referring to the development and implementation of droplet break-up models. Recent developments in droplet heating models are discussed in Section 2. It is shown that the conventional Newton's law for stationary droplet heating can be generalised to the case of transient heating via the relevant correction of the convection heat transfer coefficient. It is pointed out that in the case of realistic diesel droplet heating, the results predicted by the effective thermal conductivity model with homogeneous absorption of thermal radiation are close to those predicted by the extended model with distributed absorption of thermal radiation in droplets. The numerical model based on the analytical solution of the heat conduction equation inside droplets is recommended for implementation into computational fluid dynamics codes. Section 3 is focused on recent developments of kinetic models for droplet evaporation with a view to their application to the modelling of diesel fuel droplet evaporation into high pressure air. A new numerical algorithm for direct solution of the Boltzmann equation is applied for this analysis. The results confirm the earlier findings, that the kinetic effects are not negligible in this case and need to be taken into account in numerical modelling of the process. Results of the implementation of TAB, WAVE and stochastic models into the KIVA 2 CFD code, and preliminary comparison of the results with in-house experimental data are presented in Section 4. The main results of the paper are summarised in Section 5.

\section{Effects of droplet heating models}

The importance of the development of accurate and computer efficient models, describing fuel droplet heating and evaporation in engineering and environmental applications, is widely recognised (Sazhin, 2006) In most of these applications, the processes of droplet heating and evaporation have to be modelled alongside the effects of turbulence, combustion, droplet break-up and related phenomena in complex three-dimensional enclosures (Durand et al, 1999; Sabelnikov et al, 2006; Sazhin, 2006). This has led to a situation where finding a compromise between the complexity of the models and their computational efficiency becomes the essential precondition for successful modelling. In a series of our papers (e.g. Dombrovsky and Sazhin (2003), Sazhin et al (2004a,b), Abramzon and Sazhin (2005, 2006)), simplified models for droplet heating and evaporation have been developed. In these models sophisticated underlying physics was described using relatively simple mathematical tools. Some of these models, including those taking into account the effects of the temperature gradient inside droplets, recirculation inside them and their radiative heating, were implemented into numerical codes focused on simulating droplet convective and radiative heating, evaporation and the ignition of the fuel vapour/air mixture (Sazhin et al, 2005a,b).

During the heating and evaporation of droplets, the processes in the liquid and gas phases are, in general, closely linked. This has been demonstrated by coupled solutions of the heat conduction equation in these phases for stationary, spherical and non-evaporating droplets. Cooper (1977) solved this problem using the Laplace transformation, assuming that the gas medium is infinite. The initial body and gas temperatures were assumed to be uniform. The effects of thermal radiation were not taken into account. Sazhin et al $(2005 \mathrm{c}, \mathrm{d})$ presented the solution in the form of converging series. The size of the domain occupied by the gas was assumed to be finite, and the outer boundary of this domain was kept at constant temperature. The initial radial distribution of temperature in the 
droplet was taken into account. A modification of Newton's law for droplet heating was introduced via a correction to either the gas temperature or convection heat transfer coefficient. The results were analysed using values of parameters relevant to the problem of liquid fuel droplet heating in a diesel engine. The values of the corrections to the convection heat transfer coefficient varied from about 0.1 (large domain occupied by gas and $\mathrm{Fo}_{\mathrm{O}}=500$ ) to 2.8 (at $\mathrm{Fo}=0.1$ ). In the case of an infinitely thick spherical layer of gas, the results predicted by Sazhin et al $(2005 \mathrm{c}, \mathrm{d})$ coincide with those which follow from the formulae obtained by Cooper (1977). For sufficiently small Fo, these results coincide for any finite thickness of the gas layer. The effect of thermal radiation on droplet heating is accounted for via the additional corrections to gas temperature or the convection heat transfer coefficient. It was shown that for the values of parameters typical for diesel fuel droplets, the effects of radiation on the surface heat flux are small for small Fo, but become dominant for large Fo $(\mathrm{Fo}>50)$.

The generalisation of these solutions to the case of moving, evaporating droplets in a realistic environment, modelled by computational fluid dynamics (CFD) codes, seems infeasible at the moment. Fortunately, in many practical applications, including those in diesel engines, the diffusivity of the gas phase is substantially (more than an order of magnitude) larger than that of a liquid case. This allows us to separate the processes of these phases, taking into account that gas adjusts to changing parameters much more quickly than does liquid. The problem of heat transfer from gas to liquid using the same assumptions as in (Cooper, 1977; Sazhin et al, 2005c,d), but with fixed droplet temperature, was solved by a number of authors, including Todes (1968), Feng and Michaelides (1996) and Sazhin et al (2001). As follows from this solution, initially, the heat transfer coefficient from gas to droplets is infinitely high, but it approaches the steady state value rather rapidly. If this initial stage (a few $\mu$ in the case of diesel fuel droplet heating (Sazhin et al, 2001)) is ignored then it can be assumed that the heating of droplets by the gas phase can be described in terms of a steady-state heat transfer coefficient. This assumption is universally used in CFD codes (e.g. PHOENICS, FLUENT, KIVA, and VECTIS). It allows us to consider steady-state gas phase models, and assume that all transient processes take place only in the liquid phase. In other words we can describe droplet heating in terms of the convective heat transfer coefficient $h$.

In (Sazhin et al, 2005a) the results of implementation of the analytical solution of the heat conduction equation inside droplets, for a constant convection heat transfer coefficient $h$ (originally reported by Sazhin et al (2004b)), into a numerical codes were reported. This code was then applied to numerical modelling of fuel droplet heating and evaporation in conditions relevant to diesel engines, but without taking into account the effects of droplet break-up. This approach was shown to be more CPU effective and accurate than the approach based on the numerical solution of the discretised heat conduction equation inside the droplet, and more accurate than the solution based on the parabolic temperature profile model. The relatively small contribution of thermal radiation to droplet heating and evaporation allowed the authors to take it into account using a simplified model, which does not consider the variation of radiation absorption inside semi-transparent droplets (Sazhin et al, 2004a). This result is similar to the one reported by Abramzon and Sazhin (2005, 2006).

An approximate expression for the average radiative power absorbed per unit volume inside droplets, which turned out to be particularly useful for practical applications, was derived in the form (Dombrovsky et al, 2001; Sazhin et al, 2004a):

$$
P(R)=3 \times 10^{6} \quad \text { a } \quad \sigma \quad R_{d(\mu m)}^{b-1} \theta_{R}^{4}
$$

where $\theta_{R}$ is the radiation temperature, $R_{d(\mu \mathrm{m})}$ is the droplet radius in $\mu \mathrm{m}$, 
$a=a_{0}+a_{1} \theta_{\mathrm{R}} / 10^{3}+a_{2}\left(\theta_{\mathrm{R}} / 10^{3}\right)^{2}, b=b_{0}+a_{1} \theta_{\mathrm{R}} / 10^{3}+b_{2}\left(\theta_{\mathrm{R}} / 10^{3}\right)^{2}$,

$\theta_{R}$ can be assumed equal to the external temperature $T_{\text {ext }}$ in the case of an optically thin gas in the whole domain. Explicit expressions for the coefficients $a$ and $b$ for a typical automotive diesel fuel (e.g. low sulfur ESSO AF1313 diesel fuel) in the range of external temperatures 1000 - $3000 \mathrm{~K}$ are given in (Sazhin et al, 2004a).

In (Sazhin et al, 2004b) the solution for constant convection heat transfer coefficient $h$ was generalised to the case of almost constant $h$. In the case of arbitrary $h$ the original differential equation for heat conduction inside a droplet was reduced to the Volterra integral equation of the second kind. These results, however, were shown to be of limited practical importance for application in numerical codes (Sazhin et al, 2005a).

The model described in (Sazhin et al, 2005a) was further developed in (Sazhin et al, 2005b). In the latter paper, the effects of the temperature gradient inside fuel droplets on droplet evaporation, break-up and the ignition of fuel vapour/air mixture were investigated based on a zero-dimensional code. This code took into account the coupling between the liquid and gas phases and described the autoignition process based on the eight step chain branching reaction scheme (Shell autoignition model). The effect of the temperature gradient inside droplets was investigated by comparing the 'effective thermal conductivity' (ETC) model and the 'infinite thermal conductivity' (ITC) model, both of which were implemented into the zero-dimensional code. It was pointed out that in the absence of break-up, the influence of the temperature gradient in droplets on droplet evaporation in a realistic diesel engine environment was generally small (less than about 5\%). In the presence of the break-up process, however, the temperature gradient inside the droplets led to a significant decrease in evaporation time.

Even in the absence of break-up, the effect of the temperature gradient inside droplets led to a noticeable decrease in the total ignition delay. In the presence of break-up this effect was shown to be enhanced substantially, leading to more than a halving of the total ignition delay. It was recommended that the effect of the temperature gradient inside the droplets is taken into account in computational fluid dynamics codes describing droplet break-up and evaporation processes, and the ignition of the evaporated fuel/air mixture.

Calculations of the droplet vaporization rate performed using the simplified 'effective-conductivity' model with the internal radiation heat source uniformly distributed showed exceptionally good agreement with results obtained based on the more accurate 'extended' vaporization model with the non-uniform distribution of radiation absorption (Abramzon and Sazhin, 2005, 2006). This allowed Abramzon and Sazhin to recommend using the 'effective-conductivity' model with uniform radiation absorption for spray combustion calculations, including applications in internal combustion engines.

When modelling the heating and evaporation of a realistic polydisperse spray alongside the autoignition of the fuel vapour/air mixture, the problem of solving the system of stiff ordinary differential equations emerges. Bykov et al $(2004,2006)$ suggested a new decomposition technique for the solution of this system, based on the geometrical version of the integral manifold method (Gol'dshtein and Sobolev, 1992). This is based on comparison of the values on the right hand sides of these equations, leading to separation of the equations into equations for 'fast' and 'slow' variables. The hierarchy of the decomposition is allowed to vary with time. Equations for fast variables are solved by a stiff ODE system solver with the slow variables taken at the beginning of the time step. The solution of the equations for the slow variables is presented in a simplified form, assuming linearised variation of these variables for the known time evolution of the fast variables. This can be considered as the first order approximation for the fast manifold. This approach was 
applied to the problem of modelling heating and evaporation of polydisperse spray and the autoignition of the fuel vapour/air mixture. Its clear advantages were demonstrated from the point of view of accuracy and CPU efficiency, compared with the conventional approach widely used in CFD codes (Bykov et al, 2004, 2006; Sazhina et al, 2005). The difference between the solution of the full- and that of the decomposed-system of equations was shown to be negligibly small for practical applications. It was shown that in some cases the system of fast equations is reduced to a single equation.

\section{Kinetic effects}

Since the pioneering papers by Hertz (1882) and Knudsen (1915) the kinetic methods have been widely used in the modelling of evaporation, condensation and other transfer processes (see Sazhin (2006) and the references therein). These methods have been almost exclusively applied to the cases when gases are rarefied with Knudsen numbers exceeding $10^{-2}$. At the same time, a number of authors drew attention to the fact that even in the case where $\mathrm{Kn} \rightarrow 0$, the application of hydrodynamic methods to modelling fluid dynamics, heat and mass transfer processes is not always justified (Sone et al, 1996a,b; Takata and Aoki, 1999; Sone, 2002). In the recent paper by Kryukov et al (2004) it was demonstrated that even in the case of droplet evaporation into high pressure gas (diesel engines) the kinetic effects can be non-negligible. It was shown that these effects can lead to an increase in the evaporation time of up to 5-10\% compared with the prediction of the conventional hydrodynamic model in the case of small droplets (radii about $5 \mu \mathrm{m}$ ) injected into a hot gas with temperatures $T_{g}=750-2000 \mathrm{~K}$. The hydrodynamic models for droplet evaporation are essentially based on the assumption that molecules can be removed from the surface of droplets quickly enough to maintain fuel vapour concentration near the droplets at saturation level (Sazhin, 2006). The kinetic models take into account the finite speed of the removal of these molecules. Hence, the evaporation rate predicted by hydrodynamics models is always larger when compared with the rate predicted by kinetic models.

Although the kinetic processes take place in a very thin Knudsen layer adjacent to the droplet surface, a noticeable reduction in the density of vapour is observed between the surface of the droplet and the outer boundary of this layer. In this case, the flux density of vapour, as predicted by the kinetic model, is controlled by the vapour density at the outer boundary of the Knudsen layer. The latter is less than the vapour density at the droplet surface, which controls the vapour flux predicted by the hydrodynamic model. This is consistent with the above mentioned conclusion regarding the evaporation rates predicted by kinetic and hydrodynamic models.

The kinetic model for diesel fuel droplet evaporation used by Kryukov et al (2004) was essentially based on the analysis reported earlier by Muratova and Labuntsov (1969). This was an approximate analysis of the Boltzmann equation, performed under a number of assumptions and leading to an explicit expression for mass flux of vapour leaving the droplet. Two of these assumptions seem to be particularly important for practical applications. These are the assumption that the evaporation coefficient $\beta$ is a priori given and the assumption that no other gases, apart from vapour, are present in the Knudsen layer. Since no direct measurements or calculations of $\beta$ for diesel fuel are available the minimal and average values of this parameter for water $(0.04$ and 0.5$)$ were used.

Quantitative analysis of evaporation and condensation processes in binary mixtures seems infeasible in the approach used by Kryukov et al (2004). The application of numerical methods, such as those developed by Aristov and Tcheremissine (1992) and Bird (1994), becomes essential. This has been done by several authors and the results have been reported in a number of papers. In most of these papers, however, it was assumed that the sizes of all molecules in binary mixtures are the same. This is hardly justified in the case of diesel fuel evaporation into high pressure air, when 
the effective radius of fuel $\left(\mathrm{C}_{12} \mathrm{H}_{26}\right)$ molecules is about 2.5 larger than the effective radius of air $\left(\mathrm{N}_{2}\right)$ molecules. This was the main driving force behind the development of a new numerical algorithm, specifically designed to model the evaporation and condensation processes in binary mixtures consisting of molecules with different radii and masses (Kryukov et al, 2005). This algorithm was based on the numerical algorithm originally developed by Aristov and Tcheremissine (1992).

Shishkova and Sazhin (2006) further developed this algorithm and applied it to the same problem of evaporation of diesel fuel into high pressure air as considered by Kryukov et al (2004). The results of calculations, using this algorithm, were compared with the prediction of the hydrodynamic model and the approximate model used by Kryukov et al (2004).

The results of the comparison between the predictions of the kinetic model reported by Shishkova and Sazhin (2006) for $\beta=1$, and the hydrodynamic model for total pressure 30 bar, initial droplet temperature $300 \mathrm{~K}$, initial droplet radius $\left(R_{d 0}\right) 20 \mu \mathrm{m}$ and gas temperature $\left(T_{g}\right) 650 \mathrm{~K}$, are shown in Figure 1. The contribution of air in the Knudsen layer is taken into account. Predictions of the simplified kinetic model used by Kryukov at al (2004) are between those of the rigorous kinetic model and the hydrodynamic model. The effect of curvature of the droplets and the outer boundary of the Knudsen layer were ignored by Kryukov et al (2004), but were taken into account, when calculating mass fluxes, in the model developed by Shishkova and Sazhin (2006).

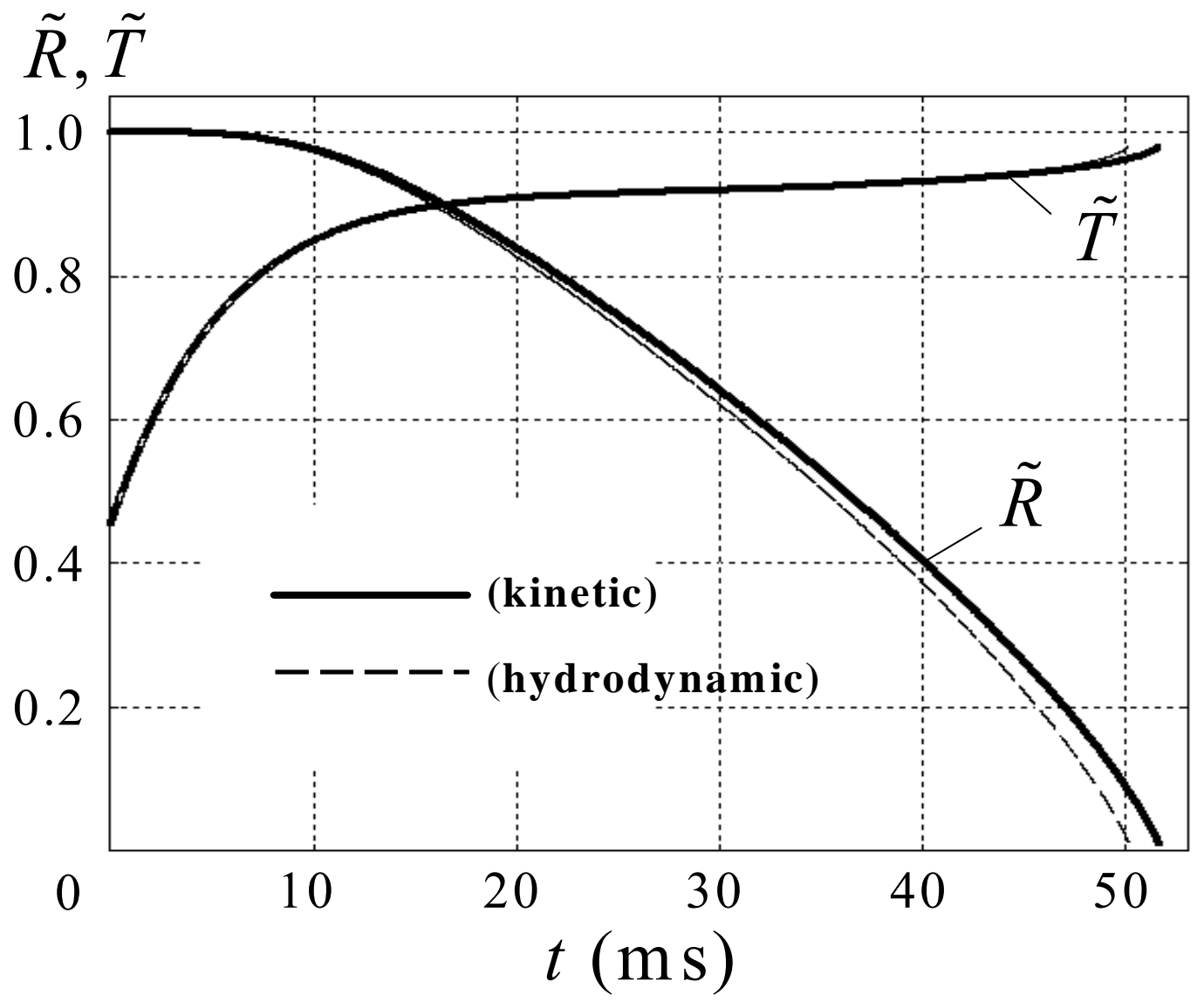

Figure 1. Plots of the normalised droplet radius $R=R_{d} / R_{d 0}$ and normalised droplet surface temperature $\stackrel{\square}{T}=T_{S} / T_{\text {cr }}$ versus time, as predicted by the rigorous kinetic model with $\beta=1$, and by the hydrodynamic model. The total pressure is $30 \mathrm{bar}$, the initial droplet temperature is $300 \mathrm{~K}, R_{d 0}=20$ $\mu \mathrm{m}$ and $T_{g}=650 \mathrm{~K}$. The contribution of air in the Knudsen layer is taken into account. The critical temperature $T_{\mathrm{cr}}=659 \mathrm{~K}$. Predictions of the simplified kinetic model used by Kryukov at al (2004) lie between the kinetic and hydrodynamic curves. 
The results of the comparison between the predictions of the models allow us to reach reliable conclusions regarding the range of applicability and accuracy of the hydrodynamic model of droplet evaporation, and the approximate kinetic model used by Kryukov et al (2004).

\section{Effects of droplet break-up models}

Since the early stages of spray modelling it has been recognised that jet and droplet break-up play a major role in spray dynamics, droplet evaporation and ultimately the autoignition of the fuel vapour/air mixture. The early break-up models were deterministic in nature, which means that the droplet break-up is presumed to be constant during the characteristic break-up time. Reitz and Diwakar's $(1986,1987)$ bag and stripping break-up models are examples of such models. These were successfully used for the analysis of spray penetration (e.g. Sazhin et al, 2003) and the effects of break-up on droplet heating (e.g. Sazhin et al, 2005b). However, the deterministic models most widely used in computational fluid dynamics (CFD) codes are the Taylor Analogy Breakup (TAB) model (O'Rourke and Amsden, 1987) and the WAVE model based on jet stability analysis (Reitz, 1987). The main limitation of the deterministic models is that they do not take into account the stochastic nature of break-up processes, which is particularly important at high Weber numbers. These effects are accounted for in stochastic break-up models (Gorokhovski, 2001; Gorokhovski and Saveliev, 2003; Apte et al, 2003) which predict explicitly the evolution of probability distribution function for the product droplets in the framework of presumed stochastic process.

The TAB, WAVE and stochastic models developed by Gorokhovski and his group have been implemented into the KIVA 2 CFD code (see Amsden, et al. (1989) for the general description of this code). Calculations of spray evolution over time were performed in two dimensions, assuming asymuthal symmetry of the flow. The spatial domain of $100 \mathrm{~mm}$ in the axial direction and $20 \mathrm{~mm}$ in the radial direction was discretised into 48 x 20 cells. Injection of parcels in the flow computational domain was performed at the axis of the nozzle exit. In total 1000 parcels were injected during the injection pulse. Injected drops were assumed to have a size equal to the nozzle diameter and axial velocity corresponding to the average injection velocity at the nozzle exit.

The results of calculations were compared with experimental data obtained on a high-pressure rapid compression machine (RCM). This machine is based around a single cylinder Ricardo Proteus test engine which was converted to two-stroke cycle operation by the addition of inlet and exhaust ports in the cylinder liner. The engine had a bore of $135 \mathrm{~mm}$, a stroke of $150 \mathrm{~mm}$, and a displacement of 2.2 litres. An optical chamber $80 \mathrm{~mm}$ in length and $50 \mathrm{~mm}$ in diameter was fitted on to the cylinder head to enable the full length of the developed fuel spray to be viewed. This chamber provided a near quiescent high-pressure environment, with realistic in-cylinder conditions being achieved by conditioning of the intake air. The size and cylindrical shape of the chamber offered sufficient space for the fuel spray to develop without wall impingement. The flow field in the chamber was quiescent to avoid disturbances by airflow motion on the spray development. The compression ratio of the engine was reduced to 9:1 to further increase the volume available for the optical chamber. In-cylinder temperatures and pressures representative of a modern engine were obtained by increasing the boost pressure and temperature. The induced air was then compressed in the engine during the compression stroke to achieve the desired test conditions.

A custom-built controller was developed (Crua, 2002) to enable independent control of injection timing, number of injections per cycle, injection duration and rail pressure. The fuel injector used was a modern electro-magnetically actuated common rail injector. The injector nozzle was a VCO type with three $200 \mu \mathrm{m}$ holes. The injection pressure was $600 \mathrm{bar}$, the in-cylinder pressure was 20 bar, and the in-cylinder gas temperature was $570 \mathrm{~K}$. Measurements of the injection rate were performed to determine the jet velocity at the nozzle outlet. 


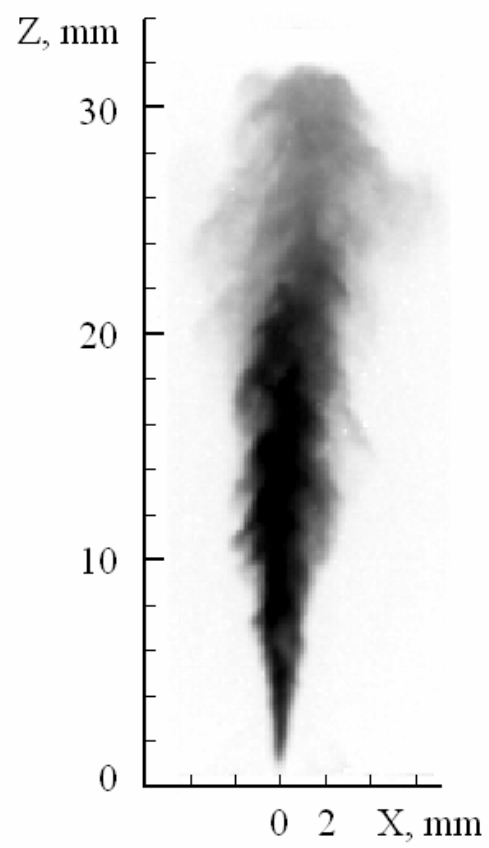

(a)

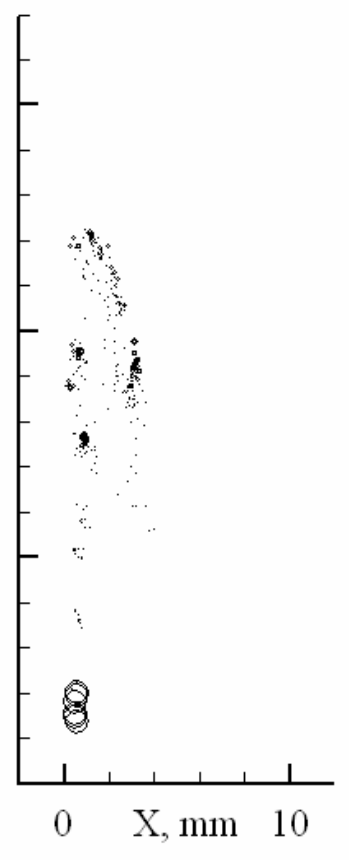

(b)

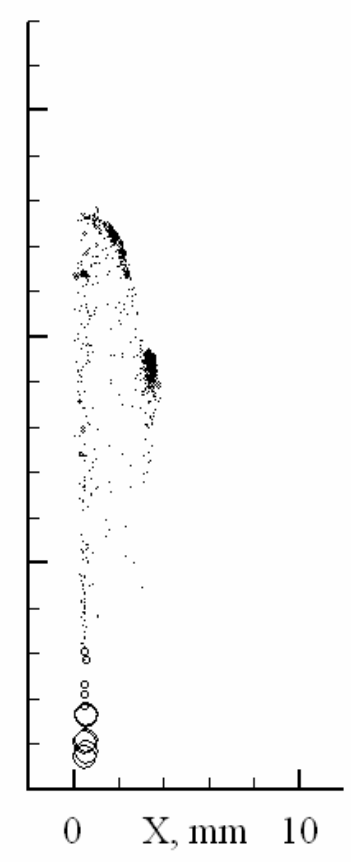

(c)

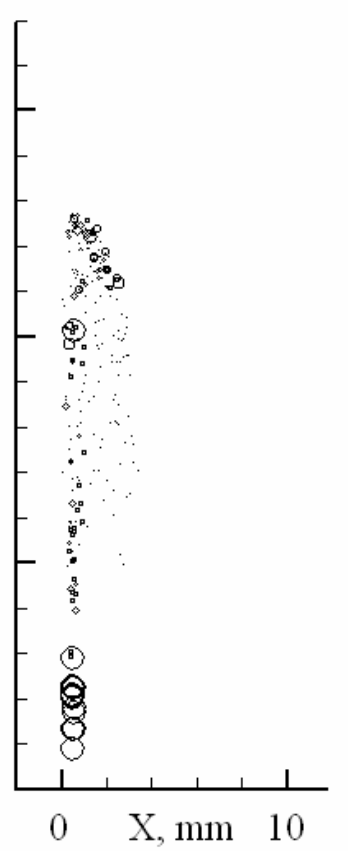

(d)

Figure 2. Patterns of diesel spray observed under the experimental conditions described above (a), predicted by the KIVA 2 CFD code using the TAB (b), WAVE (c) and stochastic (d) models of spray break-up at $t=0.5 \mathrm{~ms}$. Only the right half of the spray is shown in Figs b,c,d and scales for X and $\mathrm{Z}$ are in $\mathrm{mm}$.

Video recordings of the fuel sprays were obtained using a Phantom V7.1 high-speed video camera. The best compromise between acquisition rate and image resolution was obtained with a frame rate of $34300 \mathrm{fps}$, with a corresponding resolution of 128 × 320 pixels. Exposure time was independent of the frame rate, and set to 2 microseconds. The processing of the videos was performed by purpose-developed software that measured the spray penetration length and spray cone angle at each video frame after suitable pixel thresholds were set (Crua, 2002).

Figure 2 shows patterns of the spray predicted by the KIVA 2 CFD code using the TAB, WAVE and stochastic models, and the instantaneous image of the spray at $t=0.5 \mathrm{~ms}$. All calculations were performed assuming that the discharge coefficient of the nozzle is equal to 0.7 , and using the measured total amount of fuel, Calculations show spatial distribution of droplet parcels in the right half of the spray. As the actual size of droplets is much smaller then the scale of the figure, the droplet sizes are magnified ten times. The lengths of the spray tip penetration and the cone angles of the spray predicted by all three models are close. The comparison of the calculated spray patterns with the spray image shows larger cone angles predicted by the models. Large droplets located downstream from the nozzle exit indicate the length of the unbroken part of the spray. The stochastic model predicts wider spectra of droplets in the spray region, and longer break-up length compared to the TAB and WAVE models. Also, the TAB and WAVE models predict the fastest atomization of the spray and the smallest size of droplets at the spray tip. Large droplets in the vicinity of the spray tip appear as a result of coalescence. The largest droplets at the spray tip are predicted by the stochastic model. 


\section{Conclusions}

In the case of stationary non-evaporating droplets, a modification of Newton's law of droplet heating is introduced via a correction to either the gas temperature or convection heat transfer coefficient. These corrections were obtained from the coupled solution of the heat conduction equation in the gas and liquid phases. The solution is analysed using values of parameters relevant to the problem of a liquid fuel droplet heating in a diesel engine. Since gas diffusivity in this case is more than an order of magnitude larger than that in liquid, for practical applications in computational fluid dynamics (CFD) codes, this model can be simplified by assuming that droplet surface temperature is fixed. Moreover, if the initial stage of droplet heating (a few $\mu$ s) can be ignored then the steady-state solution for the gas phase can be applied for the analysis of the stationary processes. All transient effects in this case are accounted for by liquid phase models.

The finite thermal conductivity of the liquid, recirculation within droplets, time dependence of gas temperature and the convection heat transfer coefficient are taken into account using the effective thermal conductivity model. This model predicts results very close to those predicted by much more complicated 'extended' model. It is recommended that the numerical code, taking into account the effects of the finite thermal conductivity of the liquid and recirculation within droplets, is based on the analytical solution of the heat transfer equation inside droplets for a constant convection heat transfer coefficient. The relatively small contribution of thermal radiation to droplet heating allows its description by a simplified model, which does not consider the variation of radiation absorption inside the droplets.

A decomposition technique for the solution of the system of ODEs, describing heating and evaporation of polydisperse spray and the autoignition of the fuel vapour/air mixture, based on the geometrical version of the integral manifold method, is described. This is based on comparison of the values on the right hand sides of these equations, leading to separation of the equations into 'fast' and 'slow' variables. The hierarchy of the decomposition is allowed to vary with time. Clear advantages of this approach are demonstrated from the point of view of accuracy and CPU efficiency.

The kinetic approaches based on a simplified analysis of the Boltzmann equation and a direct solution, using a modified version of the numerical algorithm developed by Aristov and Tcheremissin, are applied to the analysis of diesel droplet evaporation into high pressure air. Kinetic models predict longer evaporation times and higher droplet temperature compared with the hydrodynamic model. These effects are more pronounced in the case of direct numerical solution of the Boltzmann equation. It is recommended that kinetic effects are taken into account when modelling the evaporation process of diesel fuel droplets in realistic internal combustion engines.

The preliminary results predicted by deterministic (TAB and WAVE) and stochastic models of droplet break-up, both implemented into the KIVA-2 code, are compared with the results of inhouse measurements of diesel sprays. The lengths of the spray tip penetration and the cone angles of the spray predicted by all three models are shown to be close. However, the stochastic approach allows us to predict a wider spectrum of droplet sizes in each spray location.

\section{Acknowledgments}

The authors are grateful to the European Regional Development Fund Franco-British INTERREG IIIa (Project Ref 162/025/247) and EPSRC (Grant EP/C527089/01) for partial financial support of the work on this paper, the EPSRC Loan Pool for supplying instruments used in this study, and their 
colleagues W Abdelghaffar, B Abramzon, V Bykov, L Dombrovsky, V Gol'dshtein, I. Goldfarb, P Krutitskii, A Kryukov and V Levashov in close collaboration with whom some original results presented in this review were obtained.

\section{References}

Abramzon, B. and Sazhin, S., 2005, Droplet Vaporization Model in the Presence of Thermal Radiation, Int J Heat Mass Transfer, $\underline{48}, 1868-1873$.

Abramzon, B. and Sazhin, S., 2006, Convective Vaporization of Fuel Droplets with Thermal Radiation Absorption, Fuel, $\underline{85}, 32-46$.

Amsden, A.A, O'Rourke, P.J, and Butler, T.D. (1989) KIVA-II: A Computer Program for Chemically Reactive Flows with Sprays. Los-Alamos National Laborat Rep. LA-11560-MS. 158 p.

Apte, S., Gorokhovski, M., and Moin, P., 2003, LES of Atomizing Spray With Stochastic Modeling of Secondary Break-up, Int. J. of Multiphase Flow, 29, 1503-1522.

Aristov, V.V. and Tcheremissine, F.G., 1992, Direct Numerical Solution of the Boltzmann Equation, Computer Centre of Russia Academy of Sciences, Moscow (in Russian).

Bird, G.A., 1994, Molecular Gas Dynamics and the Direct Simulation of Gas Flows, Oxford University Press, Oxford.

Bykov, V., Goldfarb, I., Goldshtein, V., Sazhin, S.S., Sazhina, E.M., 2004, System decomposition technique: application to spray modelling in CFD codes, 20th Annual Symposium of the Israeli Section of the Combustion Institute (Beer-Sheva), Israel, p.16.

Bykov, V., Goldfarb, I., Goldshtein, V., Sazhin, S.S., Sazhina, E.M., 2006, System decomposition technique for spray modelling in CFD codes, Computers and Fluids (in press).

Cooper, F., 1977, Heat Transfer from a Sphere to an Infinite Medium, Int. J. Heat and Mass Transfer, 20, 991-993.

Crua, C., 2002, Combustion Processes in a Diesel Engine. PhD Thesis, University of Brighton (UK).

Dombrovsky, L.A., Sazhin, S.S., Sazhina, E.M., Feng, G., Heikal, M.R., Bardsley, M.E.A., Mikhalovsky, S.V., 2001, Heating and Evaporation of Semi-transparent Diesel Fuel Droplets in the Presence of Thermal Radiation, Fuel, $\underline{80}$, 1535-1544.

Dombrovsky, L.A. and Sazhin, S.S., 2003, Absorption of Thermal Radiation in a Semi-transparent Spherical Droplet: a Simplified Model, International J of Heat and Fluid Flow, 24, 919-927.

Durand, P., Gorokhovski, M., Borghi R., 1999, An application of the Probability Function Model to Diesel Engine Combustion, Combustion Science and Technology, 144, 47-78.

Feng, Z.-G. and Michaelides, E.E., 1996, Unsteady Heat Transfer from a Sphere at Small Peclet Numbers, ASME J Fluids Engineering, 118, 96-102.

Gol'dshtein, V. and Sobolev, V., 1992, Integral manifolds in chemical kinetics and combustion, In Singularity theory and some problems of functional analysis, American Mathematical Soc., 73-92. 
Gorokhovski, M., 2001, The Stochastic Lagrangean Model of Drop Breakup in the Computation of Fuel Sprays, Atomization and Sprays, 11, 505-519.

Gorokhovski, M. and Saveliev, V.L., 2003, Analyses of Kolmogorov's Model of Breakup and its Application into Lagrangean Computation of Liquid Sprays under Air-blast Atomization, Physics of Fluids, $\underline{15}$, 184-192.

Hertz, H., 1882, Über die Verdunstung der Flussigkeiten, inbesondere des Quecksilbers, im lufteeren Raume, Ann. Phys. und Chemie, 17, 177-200.

Knudsen, M., 1915, Die Maximale Verdampfungsgeschwindigkeit des Quecksilbers, Ann. Phys., $\underline{47}, 697-708$.

Kryukov, A.P., Levashov, V.Yu. and Sazhin, S.S., 2004, Evaporation of Diesel Fuel Droplets: Kinetic Versus Hydrodynamic Models, Int J Heat Mass Transfer, 47, 2541-2549.

Kryukov, A.P., Levashov, V.Yu. and Shishkova, I.N., 2005, Condensation in the Presence of a Non-condensable Component, J. Engineering Physics and Thermophysics, 78, 15-21 (in Russian).

Muratova, T.M. and Labuntsov, D.A., 1969, Kinetic Analysis of the Evaporation and Condensation Processes, Thermal Physics of High temperatures, 7, 959-967 (in Russian).

O'Rourke, P.J. and Amsden, A.A., 1987, The TAB method for numerical calculation of spray droplet breakup, SAE Paper 872089.

Reitz, R.D., 1987, Modelling atomization processes in high-pressure vaporizing sprays, Atomisation and Spray Technology, $\underline{3}$, 309-337.

Reitz, R.D. and Diwakar, R, 1986, Effect of Drop Breakup on Fuel Sprays, SAE report 860469.

Reitz, R.D. and Diwakar, R., 1987, Structure of High-pressure Fuel Sprays, SAE report 870598.

Sabel'nikov V., Gorokhovski M., and Baricault N., 2006, The extended IEM mixing model in framework of the composition PDF approach; applications to Diesel spray combustion, Combustion Theory and Modeling, 10, 155-169.

Sazhin, S.S., 2006, Advanced Models of Fuel Droplet Heating and Evaporation, Progress in Energy and Combustion Science, $\underline{32}, 162-214$. .

Sazhin, S.S., Goldshtein, V., Heikal, M.R., 2001, A Transient Formulation of Newton's Cooling Law for Spherical Bodies, ASME J Heat Transfer, 123, 63-64.

Sazhin, S.S., Crua, C., Kennaird, D. and Heikal M.R., 2003, The Initial Stage of Fuel Spray Penetration, Fuel, $\underline{82}$, 875-885.

Sazhin, S.S., Abdelghaffar, W.A., Sazhina, E.M., Mikhalovsky, S.V., Meikle, S.T. and Bai, C., 2004a, Radiative Heating of Semi-transparent Diesel Fuel Droplets, ASME J Heat Transfer, 126, 105-109. Erratum, 2004, 126, 490-491. 
Sazhin, S.S., Krutitskii, P.A., Abdelghaffar, W.A., Sazhina, E.M., Mikhalovsky, S.V., Meikle, S.T. and Heikal, M.R., 2004b, Transient Heating of Diesel Fuel Droplets, Int J Heat Mass Transfer, 47, 3327-3340.

Sazhin, S.S., Abdelghaffar, W.A., Krutitskii, P.A., Sazhina, E.M. and Heikal, M.R., 2005a, New Approaches to Numerical Modelling of Droplet Transient Heating and Evaporation, Int J Heat Mass Transfer, $\underline{48}, 4215-4228$.

Sazhin, S.S., Abdelghaffar, W.A., Sazhina, E.M. and Heikal, M.R., 2005b, Models for Droplet Transient Heating: Effects on Droplet Evaporation, Ignition, and Break-up, Int J Thermal Science, $\underline{44}, 610-622$.

Sazhin, S.S., Abdelghaffar, W.A., Martynov, S.B., Sazhina, E.M., Heikal, M.R., Krutitskii, P.A., 2005c, Transient Heating and Evaporation of Fuel Droplets: Recent Results and Unsolved Problems. In Proceedings of '5th International Symposium on Multiphase Flow, Heat Mass Transfer and Energy Conversion’, Xi'an, China, 3-6 July 2005 (CD-ROM).

Sazhin, S.S., Krutitskii, P.A., Martynov, S.B., Mason, D., Heikal, M.R., Sazhina, E.M., 2005d, Transient Heating of a Semitransparent Droplet. In 'Proceedings of HEFAT2005 (4th International Conference on Heat Transfer, Fluid Mechanics and Thermodynamics)', Cairo, Egypt 2005; Paper number: SS1.

Sazhina, E.M., Bykov, V., Goldfarb, I., Goldshtein, V., Sazhin, S.S., and Heikal, M.R., 2005, Modelling of spray autoignition by the ODE system decomposition technique. In Proceedings of HEFAT2005 (4th International Conference on Heat Transfer, Fluid Mechanics and Thermodynamics), Cairo, Egypt; Paper number: SE2.

Shishkova, I.N. and Sazhin, S.S., 2006, A Numerical Algorithm for Kinetic Modelling of Evaporation Processes, Journal of Computational Physics (in press).

Sone, Y. Aoki, K., Takata, S., Sugimoto, H., and Bobylev, A.V., 1996a, Inappropriateness of the heat-conduction equation for description of a temperature field of a stationary gas in the continuum limit: examination by asymptotic analysis and numerical computation of the Boltzmann equation, Phys. Fluids $\underline{8}$, 628-638; Errata 1996, $\underline{8}, 841$.

Sone, Y., Takata, S., and Sugimoto H., 1996b, On the behaviour of a gas in the continuum limit in the light of kinetic theory: the case of cylindrical Couette flows with evaporation and condensation, Phys. Fluids 8, 3403-3413; Errata 1998, 10, 1239.

Sone, Y., 2002, Kinetic Theory and Fluid Dynamics, Birkhäuser, Springer.

Takata S., and Aoki, K., 1999, Two-surface problems of a multicomponent mixture of vapors and noncondensable gas, Phys Fluids 11, 2743-2756.

Todes, O.M., 1968, Quasi-stationary Regimes of Mass and Heat Transfer Between a Spherical Body and Ambient Medium. In Fedoseev V.A., editor. In 'Problems of evaporation, combustion and gas dynamics in disperse systems', Proceedings of the Sixth Conference on Evaporation, Combustion and Gas Dynamics in Disperse Systems (October 1966), Odessa: Odessa University Publishing House; pp. 151-159 (in Russian). 